\title{
High Performance Implementations of the BST Method on Hybrid CPU-GPU Platforms
}

\author{
Peter Benner \\ Max Planck Institute for Dynamics \\ of Complex Technical Systems \\ Magdeburg, Germany \\ Email: benner@mpi-magdeburg.mpg.de
}

\author{
Pablo Ezzatti \\ Instituto de Computación \\ Universidad de la República \\ Montevideo, Uruguay \\ Email: pezzatti@fing.edu.uy
}

\author{
Enrique S. Quintana-Ortí \\ and Alfredo Remón \\ Dept. de Ingeniería y \\ Ciencia de los Computadores \\ Universidad Jaime I \\ Castellón, Spain \\ Emails:\{quintana,remon\}@icc.uji.es
}

\begin{abstract}
Model order reduction is necessary in many complex scientific and engineering applications. Among the methods for model reduction, those based on the SVD are well-known for their beneficial theoretical properties, though they require $\mathbf{O}\left(n^{3}\right)$ floating-point arithmetic operations, with $n$ being in the range of $10^{3}-10^{4}$ for many practical applications. In this paper we propose several high performance implementations of the Balanced Stochastic Truncation method for model reduction. The new routines carefully distribute the computations among the computational resources of a hybrid platform composed of one (or more) multicore CPU(s) and a manycore GPU. Our results show that model reduction of a large-scale problem with 9,669 state variables, which previously required the use of a cluster of computers, can now be carried out in the target platform in less than 25 minutes.
\end{abstract}

\section{INTRODUCTION}

Model order reduction of dynamical linear systems is frequently employed to tackle the high complexity of many scientific and industrial applications [1], [2]. In particular, model order reduction can be applied to reduce the time and cost required for the design of optimal controllers in many industrial processes with the dynamics modeled by a linear time-invariant (LTI) system of the form:

$$
\begin{array}{rlrl}
\dot{x}(t) & =A x(t)+B u(t), & t>0, & x(0)=x^{0}, \\
y(t)=C x(t)+D u(t), & t \geq 0 . &
\end{array}
$$

Here, $x(t)$ contains the states of the system, $x^{0} \in \mathbb{R}^{n}$ is the initial state, $u(t) \in \mathbb{R}^{m}$ and $y(t) \in \mathbb{R}^{p}$ contain the inputs and outputs, respectively, and $A \in \mathbb{R}^{n \times n}, B \in \mathbb{R}^{n \times m}, C \in \mathbb{R}^{p \times n}$, and $D \in \mathbb{R}^{p \times m}$. In the frequency domain, the system in (1) is described by its transfer function matrix (TFM) $G(s)=$ $C\left(s I_{n}-A\right)^{-1} B+D$. In practice, the number of states (also known as the state-space dimension or the order) of the system usually satisfies $n \gg m, p$.

In model reduction the goal is to obtain a reduced-order LTI system,

$$
\begin{array}{rlrl}
\dot{\hat{x}}(t) & =\hat{A} \hat{x}(t)+\hat{B} u(t), & t>0, & \hat{x}(0)=\hat{x}^{0}, \\
\hat{y}(t)=\hat{C} \hat{x}(t)+\hat{D} u(t), & t \geq 0, &
\end{array}
$$

of order $r$, with $r \ll n$, and associated TFM $\hat{G}(s)=$ $\hat{C}\left(s I_{n}-\hat{A}\right)^{-1} \hat{B}+\hat{D}$ which approximates the dynamics of the original system defined by $G(s)$. The reduced-order realization (2) can then replace the original model of order $n$ in subsequent simulations or processes, thus simplifying these tasks considerably. Model order reduction of large-scale systems is of interest e.g., in the control and simulation of thermal, thermo-mechanical, electro-mechanical and acoustic finite element models [1]. We consider a system to be largescale if $n$ is of the order of a few thousands to a hundred of thousands, while often $m$ and $p$ are of the order of a few tens to a hundred.

In this paper, we consider the state-space truncation approach for model order reduction which requires, at an initial stage, the solution of a Lyapunov and an algebraic Riccati equation (ARE). The reduced-order system is then obtained using a variant of the balanced stochastic truncation (BST) method [3]. Other approaches for model order reduction (see, e.g., [1], [4] and the references therein) are specific for a certain subset of problems, and rarely possess relevant properties such as error bounds, preservation of stability and passivity, or phase information. A comparison of the numerical properties of SVD-based methods (e.g., BST) and Krylov subspace methods can be found in [1].

The most expensive problems from the arithmetic point of view, the solution of the Lyapunov equation and the ARE, are tackled in our algorithms via the matrix sign function, which renders a computational cost for the global model order reduction algorithm of $\mathbb{O}\left(n^{3}\right)$ flops (floating-point arithmetic operations). This requires the use of high performance computation in the reduction of models with $n$ in the order of thousands or larger.

In this work, we address the different computational stages of the BST method for model reduction of linear systems, namely, the solution of the Lyapunov equation and the ARE, the computation of the SVD, and other auxiliary computations. The target architecture is a hybrid platform consisting of one or more general-purpose multicore processors (CPUs) and a single graphics processor (GPU). We exploit these two resources by designing a hybrid numerical algorithm for model order reduction that evenly balances computations between the CPUs and the GPU. In [5] we described the acceleration of the different stages in the BST method using a GPU. Here, we extend this work in the following aspects: 
- Our new approach attains a better load balance among the CPUs and the GPU, yielding a notable reduction of the execution time.

- We propose a new implementation specific for small problems that clearly outperforms all the implementations in [5]. In particular, when applied to a model of order $n=1,357$, the execution time of BST via the new method is reduced by $25 \%$ w.r.t. the previous solution. What we should understand here by small depends on the architecture employed (see Subsection III-D).

- The GPU-based implementation described in [5] can only be applied to problems which fit in the GPU memory. Here we present a GPU-based method that is not constrained by the capacity of the GPU memory. Furthermore, this implementation reports an acceleration factor $5 \times$ w.r.t. the multicore implementation in [5] for a problem of dimension 9,699.

The rest of the paper is structured as follows. In Section II we briefly review the BST method for model order reduction, including the sign function-based Lyapunov and ARE solvers and the remaining stages of the method. In Section III we describe high performance implementations for a hybrid CPU-GPU platform. In Section IV we report experimental results that illustrate the parallelism attained by the numerical algorithms on a platform consisting of two Intel QuadCore processors connected to an NVIDIA Fermi C2050 GPU. Finally, in Section V we offer a few concluding remarks and future lines of work.

\section{Model Reduction Methods BASED ON SVD}

Relative error model reduction methods aim at minimizing $\left\|\Delta_{r}\right\|_{\infty}$ defined implicitly by $G-\hat{G}=G \Delta_{r}$. BST is a particularly popular relative error method for model reduction [6], [7], [8], but presents a high computational cost that limits the dimension of the problems it can be applied to. Using current computers, the high performance implementation provided in the Subroutine Library in Control Theory - SLICOT ${ }^{1}$ [9] can be employed to reduce models with a few thousands of statespace variables. The PLiCMR [10] library provides a messagepassing implementation for the BST method that can be used to solve larger problems, but requires a cluster of computers.

In BST methods, the reduced order model is computed truncating a balanced stochastic realization of the original model. Such a realization can be obtained as follows. Define $\Phi(s)=G(s) G^{T}(-s)$, and let $W$ be a square minimum phase right spectral factor of $\Phi$, i.e., $\Phi(s)=W^{T}(-s) W(s)$. Assume $D$ has full row rank, so that $E=D D^{T}$ is positive definite. A minimal state-space realization $\left(A_{W}, B_{W}, C_{W}, D_{W}\right)$ of $W$ is then given by (see [11], [12])

$$
\begin{array}{ll}
A_{W}=A, & B_{W}=B D^{T} \\
C_{W}=E^{-\frac{1}{2}}\left(C-B_{W}^{T} X_{W}\right), & D_{W}=E^{\frac{1}{2}}
\end{array}
$$

\footnotetext{
${ }^{1}$ Available from http://www.slicot.org.
}

Here, $W_{c}$ is the controllability Gramian of $G(s)$ given by the solution of the Lyapunov equation

$$
A W_{c}+W_{c} A^{T}+B B^{T}=0,
$$

while $W_{o}$ is the observability Gramian of $W(s)$ obtained as the stabilizing solution of the ARE

$$
\begin{aligned}
0= & \left(A-B_{W} E^{-1} C\right)^{T} W_{o}+W_{o}\left(A-B_{W} E^{-1} C\right) \\
& +W_{o} B_{W} E^{-1} B_{W}^{T} W_{o}+C^{T} E^{-1} C .
\end{aligned}
$$

Thus, the BST method requires the solution of a Lyapunov equation and an ARE. Both equations can be solved via the sign function method, as introduced in [13] and [14] respectively. In the following subsections we will detail the Lyapunov and ARE solvers implemented in this work.

\section{A. Solution of the Lyapunov equation}

The matrix sign function [14] is an efficient tool to solve stable (standard) Lyapunov equations. Several approaches have been proposed for this method. Among them, the Newton iteration for the matrix sign function in Algorithm CECLNC [13] is particularly simple and efficient, and can be employed for the solution of the Lyapunov equation in (4).

\section{Algorithm CECLNC:}

$$
\begin{aligned}
& A_{0} \leftarrow A, \tilde{S}_{0} \leftarrow B^{T} \\
& k \leftarrow 0 \\
& \text { repeat }
\end{aligned}
$$

Compute the rank-revealing QR (RRQR) decomposition

$$
\begin{aligned}
& \quad \frac{1}{\sqrt{2 c_{k}}}\left[\tilde{S}_{k}, c_{k} \tilde{S}_{k} A_{k}^{-T}\right]=Q_{s}\left[\begin{array}{c}
U_{s} \\
0
\end{array}\right] \Pi_{s} \\
& \tilde{S}_{k+1} \leftarrow U_{s} \Pi_{s} \\
& A_{k+1} \leftarrow \frac{1}{\sqrt{2}}\left(A_{k} / c_{k}+c_{k} A_{k}^{-1}\right) \\
& k \leftarrow k+1 \\
& \text { until }\left\|A_{k}-I\right\|_{1}<\tau_{l}\left\|A_{k}\right\|_{1}
\end{aligned}
$$

On convergence, after $j$ iterations, $\tilde{S}=\frac{1}{\sqrt{2}} \tilde{S}_{j}$, of dimension $\tilde{k}_{c} \times n$, is the full (row-)rank approximation of $S$, so that $W_{c}=S^{T} S \approx \tilde{S}^{T} \tilde{S}$.

The initial convergence of the Newton iteration can be accelerated using a scaling factor, e.g.,

$$
c_{k}=\sqrt{\left\|A_{k}\right\| /\left\|A_{k}^{-1}\right\|} .
$$

The number of columns of the factor $\tilde{S}$ is doubled at each iteration of the algorithm and, in consequence, the computational and storage costs associated with its update grow with the loop count. Although Algorithm CECLNC presents an asympthotic quadratic convergence, and hence, a moderate number of iterations is expected, a strategy to reduce this growth is necessary. For this purpose, an RRQR factorization is computed at each step, obtained, e.g., by means of the traditional QR factorization with column pivoting [15] plus a reliable rank estimator. For simplicity, we do not detail this compression procedure. 
In the convergence test, $\tau_{l}$ is a tolerance threshold for the iteration that is usually set as a function of the problem dimension and the machine precision $\varepsilon$. In particular, to avoid stagnation of the iteration, we fix $\tau_{l}=n \cdot \sqrt{\varepsilon}$ and perform one or two additional iteration steps after the stopping criterion is satisfied. Due to the quadratic convergence of the Newton iteration, this is usually enough to reach the attainable accuracy.

\section{B. Solution of the algebraic Riccati equation}

The matrix sign function can also be employed to solve an ARE of the form

$$
F^{T} X+X F-X G X+Q=0 .
$$

The solution to this equation can be obtained from the stable invariant subspace of the Hamiltonian matrix [16]

$$
H=\left[\begin{array}{cc}
F & G \\
-Q & -F^{T}
\end{array}\right] .
$$

To do so, the matrix sign function of $H$ is first computed [14]:

$$
\operatorname{sign}(H)=Y=\left[\begin{array}{ll}
Y_{11} & Y_{12} \\
Y_{21} & Y_{22}
\end{array}\right],
$$

and then, the overdetermined, but consistent linear system

$$
\left[\begin{array}{c}
Y_{11} \\
Y_{12}+I_{n}
\end{array}\right] X=\left[\begin{array}{c}
I_{n}-Y_{21} \\
-Y_{11}
\end{array}\right]
$$

is solved (e.g., applying the least squares method).

The steps required by this method are summarized in Algorithm GECRSG.

$$
\begin{aligned}
& \text { Algorithm GECRSG: } \\
& H_{0} \leftarrow\left[\begin{array}{cc}
F & G \\
-Q & -F^{T}
\end{array}\right] \\
& k \leftarrow 0 \\
& \text { repeat } \\
& \quad H_{k+1} \leftarrow \frac{1}{2}\left(H_{k} / d_{k}+d_{k} H_{k}^{-1}\right) \\
& \text { until }\left\|H_{k+1}-H_{k}\right\|_{1}<\tau_{r}\left\|H_{k}\right\|_{1}
\end{aligned}
$$

Solve

$$
\left[\begin{array}{c}
Y_{11} \\
Y_{12}+I_{n}
\end{array}\right] X=\left[\begin{array}{c}
I_{n}-Y_{21} \\
-Y_{11}
\end{array}\right]
$$

Note that the scaling factor $d_{k}$ and the tolerance threshold $\tau_{r}$ can be defined here following the ideas presented in the previous subsection for $c_{k}$ and $\tau_{l}$, respectively.

\section{HIGH PERFORMANCE IMPLEMENTATION ON A HYBRID ARCHITECTURE}

In this section, we describe several implementations of the BST model order reduction method on a hybrid architecture composed of one or more general-purpose multicore processors connected to a GPU. In the next subsections, we will expose the parallel implementations for the different stages of the algorithm, focusing on the most time consuming parts, namely the Lyapunov and ARE solvers. Additionally, we provide some hints on how to select a globally efficient implementation which depends on the problem size and the hardware employed.

\section{A. Implementations of the Lyapunov solver}

Algorithm CECLNC for the solution of Lyapunov equations requires $O\left(n^{3}\right)$ flops per iteration, most of them necessary for the computation of the inverse matrices $A_{k}^{-1}$. In fact, this operation involves itself $2 n^{3}+O\left(n^{2}\right)$ flops. To exploit the resources available on the target architecture, we propose three different implementations for this algorithm.

1) Multicore implementation: This code employs high performance multi-threaded implementations of linear algebra kernels from BLAS and LAPACK, as provided e.g. by the Intel MKL library. Those kernels are employed to compute the main operations present in Algorithm CECLNC, such as the matrix inversion or the $\mathrm{QR}$ decomposition. Other minor operations are parallelized using OpenMP directives, but have a negligible impact on the execution time of the solver.

2) Hybrid implementation: The objective of this routine is to execute each operation in the most appropriate device.

Our hybrid implementation proceeds as follows: The RRQR decomposition is executed using BLAS and LAPACK routines. The update of $\tilde{S}_{k+1}$ is parallelized using OpenMP directives. Then, CPU and GPU collaborate in the computation of $A_{k+1}^{-1}$. Finally, a single loop, parallelized employing OpenMP directives, computes simultaneously $A_{k+1}$ and the norms required by the loop guard.

The massively parallel architecture of the GPU has been demonstrated to deliver high performance for the execution of large linear algebra kernels [17]. Thus, these devices can be used to accelerate the computation of the large operations that appear in Algorithm CECLNC. The most expensive computations present in the algorithm correspond to the inversion of matrices $A_{k}$. In this implementation, the $\mathrm{CPU}(\mathrm{s})$ and the GPU cooperate in the computation of each inverse. During the computation of $A_{k}^{-1}$, fine-grain operations are executed in the CPU, while the bulk of computations, namely the matrixmatrix products, are performed in the GPU.

The matrix inversion kernel employed in this implementation is described in [18] and applies several optimization techniques: a blockwise structure to exploit the hierarchical organization of the memory, hybrid and concurrent computing $(\mathrm{CPU}+\mathrm{GPU})$ to increment the resource utilization, look-ahead techniques [19] to avoid bottlenecks, padding to accelerate the memory accesses on the GPU, and multilevel blocks strategies to improve throughput of both devices.

The remaining operations are computationally less expensive and/or present a fine-grain parallelism which renders them unattractive for a massively parallel architecture like a GPU. For this reason, and in order to reduce overheads intrinsic to data transfers, those operations are all performed in the CPU.

3) Balanced hybrid implementation: The novel balanced hybrid implementation of Algorithm CECLNC differs from the 


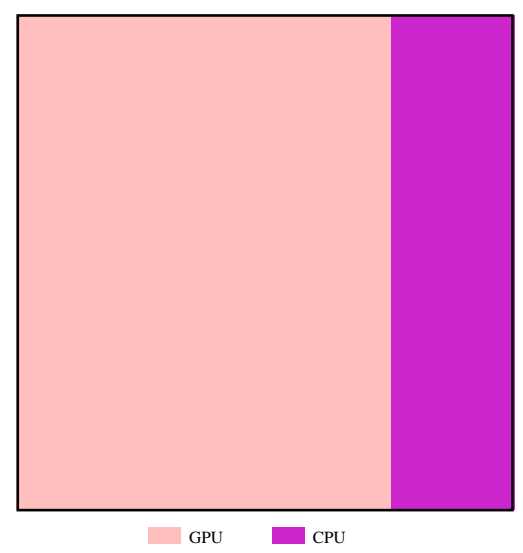

Figure 1. Data distribution between the CPU and the GPU for the balanced hybrid implementation.

previous one in the way the inverses are computed. In this variant these operations are performed as follows: Initially, the matrix $A_{k}$ is partitioned into two column panels, see Figure 1; elements in the left-hand side column panel are transferred to the GPU, where they are stored and updated during the algorithm. Elements in the right-hand side column panel are stored and updated in the CPU. The optimal sizes of both column panels depend on the matrix dimension and the computational power of the CPU and the GPU employed. An iterative process is then initiated. During this process, both architectures cooperate in computing $A_{k}^{-1}$. At a given iteration, the GPU updates the elements placed in the left column panel, while the CPU updates the elements in the right column panel and performs some fine grain operations, i.e., computes pivoting.

The benefits from this new approach can be summarized as follows:

- The most relevant is that, as the whole matrix does not need to be stored in the GPU memory, the amount of memory in the GPU is no longer a limiting factor.

- At the same time, load can be balanced between the CPU and the GPU by carefully selecting the dimension of the matrix partitioning, so as to maximize the utilization of resources.

- The padding technique necessary to obtain high performance in the GPU can be easily replaced by an appropriate matrix partitioning, fixing the number of columns in the GPU column panel to be a multiple of 32. This avoids unnecessary flops associated with the padding.

- The amount of required data transfers is decreased.

The performance attained with this implementation strongly depends on the size of the matrix partitioning. An optimal division will maximize resource utilization and minimize execution time. Two basic strategies can provide a fair partitioning: first, the number of columns stored in the GPU memory should be a multiple of 32; second, the sizes from both column panels should be proportional to the difference between the peak performance of the CPU and the GPU. Thus, if the GPU

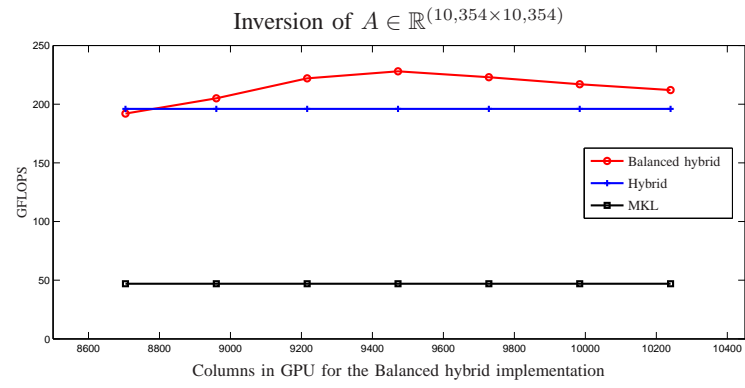

Figure 2. Inversion of a matrix $\in \mathbb{R}^{(10,354 \times 10,354)}$ using MKL, the hybrid implementation and the new balanced hybrid implementation with different matrix partitions.

peak performance is ten times higher than that of the CPU, good results will be obtained if the GPU column panel is ten times larger than the CPU column panel. Figure 2 shows the results (resulting from example STEEL_I $I_{5177}$ described in Section IV) obtained for the inversion of a matrix of dimension 10,354 on a platform where the GPU peak performance is approximately ten times that of the CPU. The new balanced hybrid implementation is the fastest, being $4 \times$ faster than the MKL implementation and a $15 \%$ faster than our original hybrid implementation. Moreover, the best performance is obtained when, approximately, $90 \%$ of the columns are updated in the GPU, Thus, the CPU-GPU performance ratio is similar to the ratio of panel sizes. There is an exception to this rule: when the dimension of the matrix $A_{k}$ is small, the best option is to compute the inverse completely in the CPU. This is due to the overhead introduced by data transfers.

\section{B. Implementations of the ARE solver}

In this subsection we present three efficient implementations of Algorithm CECRSG, a multicore implementation and two hybrid alternatives.

Algorithm CECRSG can be divided into three steps:

- First, it is necessary to build the matrix $H$ by performing some matrix-matrix multiplications (see equations (3)(5)).

- Second, the sign function for the extended matrix (7) is computed.

- Finally, the overdetermined system in (9) is solved.

Here, we should remark that the dimension of matrix $H$ is twice as big as the dimension of matrix $A$ and, consequently, the computational cost of the ARE solver is considerably higher than that of the Lyapunov solver.

1) Multicore implementation: In this code, all the computations are performed by the CPU. Whenever possible, BLAS and LAPACK kernels are employed; this is the case for the most time consuming operations (matrix-matrix products, matrix inversion,...). Some other minor operations, like the matrix addition, are accelerated using OpenMP directives.

2) Hybrid implementation: In this implementation, CPU and GPU collaborate to perform the work, but each operation is executed in the most convenient device while simultaneously trying to reduce the overhead introduced by data transfers. 
First, the matrix $H$ is built. This step mainly requires memory copies and, thus, the associated computational cost is moderate. For this reason, it is executed in the CPU, and hence no CPU-GPU data transfers are required. Then, the sign function of $H$ is computed. As previously discussed, the cost of this operation is dominated by the inversion of $H_{k}$, and can be efficiently computed using the hybrid implementation in [18].

3) Balanced hybrid implementation: Our third implementation differs from the previous one in the way $H_{k}^{-1}$ is computed. Here we employ the balanced hybrid implementation presented for the solution of the Lyapunov equation, which is even more justified due to the large dimension of the matrix. The dimension of the problems that can be tackled with the hybrid implementation is limited by the size of the GPU memory. Therefore, we propose to use the novel version where a set of columns are updated in the CPU and, consequently, the GPU memory is no longer a limiting factor.

\section{Remaining stages of the BST method}

Once the low rank factors for the controllability Gramian $\left(W_{c}\right)$ and the observability Gramian $\left(W_{o}\right)$ have been computed from the solution of the Lyapunov and the Riccati equations, respectively, only some minor operations with moderate computational effort are required to obtain the reduced order model.

The main computations in this step include a few matrixmatrix products, involving matrices of relatively small dimension. Thus, all these operations require a moderate number of arithmetic operations. Therefore, they can be efficiently computed by the CPU using BLAS, and doing so avoids data transfers and the associated overhead.

\section{Selecting the best choice}

We have described several parallel codes for the solution of the Lyapunov equation and the ARE; we now provide some hints on how to select the best combination in order to minimize the total execution time of the BST method. This will basically depend on the size of the problem to solve.

- Small to medium problems. The GPU will not deliver high performance when targeting small problems. On the other hand, the multicore implementation will perform well and avoid data transfer overheads. Thus, we recommend the multicore implementation of the Lyapunov solver. For the solution of the ARE, we recommend the hybrid approach. The matrix is twice as large as that involved in the Lyapunov equation, so that the use of the GPU is in general justified.

- Medium to large problems. In this case, the use of the GPU will accelerate the solution of the Lyapunov equation, whether using the hybrid or the balanced hybrid implementation. For the ARE solver, as the matrix involved is larger, we recommend the balanced hybrid implementation. We can fix the matrix partition of $H$ depending on the peak performances of the target CPU and the GPU. As previously discussed, good performance is obtained with a partition where the ratio defined by the dimensions of the CPU and the GPU column panels is similar to the CPU-GPU computational power ratio (see Subsection III-A).

Note that the exact meaning of small, medium and large will depend on the specifications of the target platform such as the computational power provided by the CPU and the GPU, the GPU memory availability, and the CPU-GPU interconnection bandwidth. For example, in the platform employed in our experiments (see Section IV), we can consider a problem to be small if its state-space dimension, $\mathrm{n}$, is up to 2,000; while the dimension of a larger problem up to 3,500 should be considered as a medium.

\section{NUMERICAL EXPERIMENTS}

In this section we present experimental results obtained in a platform consisting of two INTEL Xeon QuadCore E5410 processors $(2.33 \mathrm{GHz})$ with $8 \mathrm{~GB}$ of RAM connected to an Nvidia Fermi C2050 via a PCI-e (2.0) bus. The implementations employ kernels from the high performance linear algebra libraries BLAS and LAPACK, in particular, from the multithreaded INTEL MKL library (version 10.2) and the NVIDIA CUBLAS library (version 3.2) for the CPU and the GPU, respectively. Additionally, some minor operations are parallelized via OpenMP directives.

Five implementations are compared in this section:

- BST_Seq: A sequential implementation where all the computations are performed using a single Intel core.

- BST_MC: A multicore implementation fully executed in the CPU.

- BST_Hs: A hybrid implementation for small problems which solves the Lyapunov equation in the CPU while the CPU and the GPU cooperate for the solution of the ARE.

- BST_Hm: A hybrid implementation, where the CPU and the GPU cooperate in the solution of the Lyapunov equations and the ARE. This version employs the hybrid implementation for the solution of the Lyapunov equation, and the balanced hybrid implementation for the solution of the ARE.

- BST_HI: An alternative hybrid implementation where the CPU and the GPU cooperate in the solution of the Lyapunov equation and the ARE; in this variant, though, the balanced hybrid implementations of both solvers are employed. Note that this implementation is not limited by the size of the GPU memory.

We employ double precision arithmetic for the solution of three benchmarks from two model reduction problems extracted from the Oberwolfach benchmark collection ${ }^{2}$ (University of Freiburg):

- STEEL_I: this problem [20] arises in the manufacturing process of steel profiles. The goal is to design a control that yields moderate temperature gradients when the rail is cooled down. The mathematical model corresponds to the boundary control for a 2-D heat equation. A finite

\footnotetext{
${ }^{2}$ http://www.imtek.de/simulation/benchmark/.
} 
element discretization, followed by adaptive refinement of the mesh, results in the example in this benchmark. The problem dimensions depend of the discretization mesh; the two versions employed are STEEL_I $I_{1357}$ with $n=$ 1,357 and STEEL_I $I_{5177}$ with $n=5,177$, where in both cases, $m=7, p=6$.

- FLOW_METER: this is a 2-D model of an anemometerlike structure mainly consisting of a tube and a small heat source. The Dirichlet boundary conditions are applied to the original system. The reference temperature is set to $300 \mathrm{~K}$, and Dirichlet boundary conditions as well as initial conditions are set to 0 with respect to the reference. The dimensions of this problem are $n=9,669, m=1$, $p=5$.

Table I summarizes the results (in seconds) obtained for the three benchmarks evaluated. All the results in the table include the costs to perform all the necessary CPU-GPU data transfers. Column 2 shows the time dedicated to solve the Lyapunov equation; columns 3 and 4 report the time required to build the matrix $H$ and to compute $\operatorname{sign}(H)$, respectively; column 5 displays the time required to solve the overdeterminated system. Some results are omitted in this table, in particular, results of the BST_HI implementation are only given for the FLOW_METER problem, because the other two benchmarks fit into the GPU memory and, in such a case, BST_Hm is more efficient. In the FLOW_METER problem, results for the hybrid approaches BST_HS and BST_Hm are not shown, since they cannot be applied to this case due to memory limitations.

The fastest implementation for STEEL_I 1357 is BST_Hs. This implementation combines the fastest codes for the solution of the Lyapunov equation and the ARE. The small dimension of those problems yields no gain from the use of the GPU architecture for the solution of the Lyapunov equation. In this particular problem, the hybrid Lyapunov solver is nearly $6 \times$ slower than the multicore approach. This is mainly a consequence of the CPU-GPU data transfers overhead, the required synchronization points, and the non efficient use of the GPU due to the small problem dimension. However, as the dimension of the matrix $H$ is twice as large, the GPU accelerates the solution of the ARE by a factor of $2 \times$. In general, when the amount of computations required is large enough, the CPU-GPU communication overhead is compensated by exploiting the higher computational power of the GPU.

The hybrid implementations provide the best performance for the STEEL_I $I_{5177}$ benchmark. There is a notorious improvement when the GPU is used for the solution of both equations. The Lyapunov solver is accelerated by a factor $2 \times$, while the ARE solver is accelerated by a factor $4 \times$ when using the hybrid implementation, and a factor $5 \times$ when using the balanced hybrid implementation. Consequently, the BST_HI is the most efficient implementation.

Finally, the results for the last benchmark, FLOW_METER, confirm the remarkable acceleration obtained when part of the computation is off-loaded to the GPU. The solution of both equations is drastically accelerated using the proposed balanced hybrid implementation. Particularly, the Lyapunov solver is accelerated by a factor $5 \times$, while the ARE solver is nearly $6 \times$ faster than the multicore implementation. Note also that this implementation allows the use of the GPU for the solution of larger problems, avoiding the memory limitations present in implementations BST_Hs and BST_Hm.

In all the cases, most of the time is dedicated to the solution of the ARE, in particular the computation of $\operatorname{sign}(H)$ (column 4). The rest of the time is basically spent in the Lyapunov equation solver. A careful study of these two operations demonstrates that the computational effort is concentrated in the calculation of matrix inverses. This operation is notably accelerated in the proposed implementation using parallel codes and, in some cases, off-loading the major part of the computations to the GPU.

\section{CONCLUding REMARKS}

We have presented three hybrid high performance parallel implementations for model reduction via the BST method which attain remarkable efficiencies, outperforming the results reported in [5]. Variant BST_Hs is optimized for the solution of small problems, off-loading computations to the GPU only for the most time consuming stage, the solution of the ARE. Variant BST_Hm obtains the best performance for mediumsized problems, reporting important gains from the use of the GPU for the solution of the Lyapunov equation and the ARE. However, the applicability of this implementation is limited by the GPU memory. Variant BST_HI includes a new code for matrix inversion which efficiently distributes computations among CPU and GPU, increasing the resource usage and reducing the execution time. In addition, it allows the solution of problems that do not fit in the GPU memory.

All the implementations obtain high performance in the hybrid target platform, exploiting the resources present in the platform and the main features of the multicore CPU(s) and the GPU. This is achieved using an intelligent task scheduling and two levels of parallelism: multi-threaded linear algebra kernels included in the BLAS libraries (MKL for the CPU and CUBLAS for the GPU) are employed to perform the most time-consuming linear algebra operations; however, whenever possible, operations proceed concurrently in both architectures, overlapping computations in the CPU and the GPU.

Experimental results on a platform consisting of a stateof-the-art general-purpose multicore architecture and a Fermi GPU show that model order reduction of large-scale linear systems can be significantly accelerated using this class of platforms.

Future work will include:

- Exploiting the use of multiple GPUs to further reduce the computational time.

- Test linear algebra kernels from other libraries recently developed. 


\begin{tabular}{|c|c|c|c|c|c|}
\hline Implementation & $\begin{array}{r}\text { Lyapunov } \\
\text { solver }\end{array}$ & Build $H$ & $\operatorname{sign}(H)$ & $\begin{array}{r}\text { Overdeterminated } \\
\text { system solve }\end{array}$ & $\begin{array}{c}\text { Total } \\
\text { time(s) }\end{array}$ \\
\hline \multicolumn{6}{|c|}{ STEEL_I 1357} \\
\hline BST_Seq & 7.7 & 0.10 & 118.1 & 3.2 & 129.1 \\
\hline BST_MC & 1.6 & 0.05 & 22.3 & 0.5 & 24.5 \\
\hline BST_HS & 1.6 & 0.05 & 10.9 & 0.5 & 13.1 \\
\hline BST_Hm & 9.4 & 0.05 & 10.9 & 0.5 & 20.9 \\
\hline \multicolumn{6}{|c|}{ STEEL_I 5177} \\
\hline BST_Seq & 334.1 & 1.5 & 6404.6 & 325.3 & 7065.6 \\
\hline BST_MC & 63.7 & 0.8 & 1127.8 & 24.9 & 1217.2 \\
\hline BST_HS & 63.7 & 0.8 & 292.9 & 24.9 & 382.3 \\
\hline BST_Hm & 26.8 & 0.8 & 292.9 & 24.9 & 345.4 \\
\hline BST_HI & 26.8 & 0.8 & 232.8 & 24.9 & 285.3 \\
\hline \multicolumn{6}{|c|}{ FLOW_METER } \\
\hline BST_Seq & 2303.4 & 7.1 & 43103.3 & 1035.5 & 46449.3 \\
\hline $\mathrm{BST} \_\mathrm{MC}$ & 376.8 & 3.4 & 7105.3 & 150.1 & 7635.6 \\
\hline BST_HI & 78.8 & 3.4 & 1232.1 & 150.1 & 1464.4 \\
\hline
\end{tabular}

Table I

EXECUTION TIME (IN SECS.) OF THE DIFFERENT STEPS OF THE BST METHOD FOR THE STEEL_I AND THE FLOW_METER PROBLEMS.

\section{ACKNOWLEDGMENTS}

The researchers from the UJI were supported by project TIN2011-23283 and FEDER; and project P1B-2011-18 of the Fundación Caixa-Castellón/Bancaixa and UJI.

\section{REFERENCES}

[1] A. Antoulas, Approximation of Large-Scale Dynamical Systems. Philadelphia, PA: SIAM Publications, 2005.

[2] P. Benner, V. Mehrmann, and D. Sorensen, Eds., Dimension Reduction of Large-Scale Systems, ser. Lecture Notes in Computational Science and Engineering. Springer-Verlag, Berlin/Heidelberg, Germany, 2005 , vol. 45 .

[3] P. Benner, E. S. Quintana-Ortí, and G. Quintana-Ortí, "Efficient numerical algorithms for balanced stochastic truncation," Internat. J. in Applied Mathematics and Computer Science, vol. 11, no. 5, pp. 11231150, 2001.

[4] R. Freund, "Reduced-order modeling techniques based on Krylov subspaces and their use in circuit simulation," in Applied and Computational Control, Signals, and Circuits, B. Datta, Ed. Boston, MA: Birkhäuser, 1999, vol. 1, ch. 9, pp. 435-498.

[5] P. Benner, P. Ezzatti, E. S. Quintana-Ortí, and A. Remón, "Accelerating BST methods for model reduction with graphics processors," in 9th International Conference on Parallel Processing and Applied Mathematics, 2011, to appear.

[6] U. Desai and D. Pal, "A transformation approach to stochastic model reduction," IEEE Trans. Automat. Control, vol. AC-29, pp. 1097-1100, 1984.

[7] M. Green, "Balanced stochastic realization," Linear Algebra Appl., vol. 98, pp. 211-247, 1988

[8] A. Varga and K. H. Fasol, "A new square-root balancing-free stochastic truncation model reduction algorithm," in Prepr. 12th IFAC World Congress, vol. 7, Sydney, Australia, 1993, pp. 153-156.

[9] A. Varga, "Task II.B.1 - selection of software for controller reduction," The Working Group on Software (WGS), SLICOT Working Note 199918, December 1999, available from http://www.slicot.org.
[10] P. Benner, E. Quintana-Ortí, and G. Quintana-Ortí, "State-space truncation methods for parallel model reduction of large-scale systems," Parallel Comput., vol. 29, pp. 1701-1722, 2003.

[11] B. D. O. Anderson, "An algebraic solution to the spectral factorization problem," IEEE Trans. Automat. Control, vol. AC-12, pp. 410-414, 1967.

[12] B. D. O. Anderson., "A system theory criterion for positive real matrices," SIAM J. Cont., vol. 5, pp. 171-182, 1967.

[13] P. Benner, E. Quintana-Ortí, and G. Quintana-Ortí, "Solving linearquadratic optimal control problems on parallel computers," Optimization Methods Software, vol. 23, no. 6, pp. 879-909, 2008.

[14] J. Roberts, "Linear model reduction and solution of the algebraic Riccati equation by use of the sign function," Internat. J. Control, vol. 32, pp. 677-687, 1980, (Reprint of Technical Report No. TR-13, CUED/BControl, Cambridge University, Engineering Department, 1971).

[15] G. Golub and C. Van Loan, Matrix Computations, 3rd ed. Baltimore: Johns Hopkins University Press, 1996.

[16] P. Benner, R. Byers, E. Quintana-Ortí, and G. Quintana-Ortí, "Solving algebraic Riccati equations on parallel computers using Newton's method with exact line search," Parallel Comput., vol. 26, no. 10, pp. $1345-1368,2000$

[17] S. Barrachina, M. Castillo, F. D. Igual, R. Mayo, E. S. Quintana-Ortí, and G. Quintana-Ortí, "Exploiting the capabilities of modern GPUs for dense matrix computations," Concurrency and Computation: Practice and Experience, vol. 21, pp. 2457-2477, 2009.

[18] P. Ezzatti, E. Quintana-Ortí, and A. Remón, "Using graphics processors to accelerate the computation of the matrix inverse," The Journal of Supercomputing, vol. 58, pp. 429-437, 2011.

[19] P. Strazdins, "A comparison of lookahead and algorithmic blocking techniques for parallel matrix factorization," Department of Computer Science, The Australian National University, Canberra 0200 ACT, Australia, Tech. Rep. TR-CS-98-07, 1998.

[20] P. Benner and J. Saak, "A semi-discretized heat transfer model for optimal cooling of steel profiles," in Dimension Reduction of Large-Scale Systems, ser. Lecture Notes in Computational Science and Engineering, P. Benner, V. Mehrmann, and D. Sorensen, Eds. Springer-Verlag, Berlin/Heidelberg, Germany, 2005. 